\title{
De Haas-van Alphen effect in metals without inversion center
}

\author{
V. P. Mineev ${ }^{(1)}$ and K. V. Samokhin ${ }^{(2)}$ \\ (1) Commissariat á l'Energie Atomique, DSM/DRFMC/SPSMS 38054 Grenoble, France \\ (2) Department of Physics, Brock University, St.Catharines, Ontario, Canada L2S $3 A 1$
}

(Dated: March 24, 2005)

\begin{abstract}
We show how the de Haas-van Alphen effect can be used to directly measure the magnitude of spin-orbit coupling in non-centrosymmetric metals, such as $\mathrm{CePt}_{3} \mathrm{Si}$ and $\mathrm{LaPt}_{3} \mathrm{Si}$.

PACS numbers: 71.18.+y, 74.70.Tx
\end{abstract}

The recent discovery of superconductivity in a non-centrosymmetric heavy-fermion compound $\mathrm{CePt}_{3} \mathrm{Si}^{\underline{1}}$ has renewed interest, both experimenta12.3.4 and theoretical,,$\frac{5,6,7,8,9,10,11,12}{10}$ to such materials. A peculiar property of non-centrosymmetric metals is that the spinorbit coupling plays an essential role in the formation of single-electron states, namely it leads to the splitting of the energy bands characterized by helicity (i.e. the spin projection on the direction of momentum). This has important consequences for superconductivity: the electrons with opposite momenta have the same energies only if they are from the same non-degenerate band. For electrons from different bands this is possible only at some degeneracy lines or points in momentum space. Therefore, a large enough band splitting prevents the Cooper pairing of electrons from different bands.

Theoretically, the magnitude of the band splitting can be determined from the band structure calculations. On the other hand, one can obtain some experimental information about it from the frequencies of de Haasvan Alphen ( $\mathrm{dHvA}$ ) oscillations of magnetization. The first $\mathrm{dHvA}$ measurements in non-centrosymmetric metals have been reported in Ref. 13. While the restoration of the Fermi surface in $\mathrm{CePt}_{3} \mathrm{Si}$ is difficult due to large values of the effective masses, the measurements on its light-electron counterpart $\mathrm{LaPt}_{3} \mathrm{Si}$ have revealed rich information about the band structure.

Previous experimental work on the $\mathrm{dHvA}$ and a closely related Shubnikov-de Haas effects in systems without inversion center focused either on asymmetric semiconductor heterostructures $14,15,16$ or on bulk semiconductors with zinc-blende structure 17 A common feature of these systems is that the spin-orbit band splitting results in two distinct frequencies of the $\mathrm{dHvA}$ oscillations. When the frequencies are close, their interference produces a characteristic beating pattern in the observed signal. This phenomenon was first theoretically predicted in Ref. 18 (for recent work on the subject, see e.g. Ref. 19). Analyzing the beating pattern allows one to estimate the strength of the spin-orbit coupling. In this brief article we apply these ideas to the interpretation of the dHvA data in $\mathrm{CePt}_{3} \mathrm{Si}$ and $\mathrm{LaPt}_{3} \mathrm{Si}$.

The effective single-electron Hamiltonian in a noncentrosymmetric crystal can be written in the form

$$
H=\epsilon_{0}(\boldsymbol{k})+\boldsymbol{\alpha}(\boldsymbol{k}) \boldsymbol{\sigma}-\mu_{B} \boldsymbol{H} \boldsymbol{\sigma},
$$

where $\epsilon_{0}(\boldsymbol{k})$ is the band energy, the spin-orbit coupling is described by a pseudovector function $\boldsymbol{\alpha}(\boldsymbol{k})=-\boldsymbol{\alpha}(-\boldsymbol{k})$, and $\boldsymbol{\sigma}=\left(\sigma_{x}, \sigma_{y}, \sigma_{z}\right)$ is the vector composed of Pauli matrices. The last term describes the Zeeman interaction with an external magnetic field $\boldsymbol{H}$, with $\mu_{B}$ being the Bohr magneton [using a general form of the Zeeman energy for band electrons, $\mu_{i j}(\boldsymbol{k}) H_{i} \sigma_{j}$, would not add anything to the substance of our results]. The orbital effect of the field can be included by replacing $\boldsymbol{k} \rightarrow \boldsymbol{k}+(e / \hbar c) \boldsymbol{A}(\hat{\boldsymbol{r}}){ }^{20}$ where $\hat{\boldsymbol{r}}=i \boldsymbol{\nabla}_{\boldsymbol{k}}$ is the position operator in the $\boldsymbol{k}$-representation and $e$ is the absolute value of the electron charge.

The momentum dependence of the pseudovector $\boldsymbol{\alpha}(\boldsymbol{k})$ is determined by the point symmetry of the crystal. In the case of the tetragonal group $\mathbf{C}_{4 v}$, which describes the symmetry of both $\mathrm{CePt}_{3} \mathrm{Si}$ and $\mathrm{LaPt}_{3} \mathrm{Si}$, it can be written quite generally in the form $\boldsymbol{\alpha}(\boldsymbol{k})=\alpha_{\perp}\left[\boldsymbol{\varphi}_{E}(\boldsymbol{k}) \times\right.$ $\hat{z}]+\alpha_{z} \varphi_{A_{2}}(\boldsymbol{k}) \hat{z}$, where $\boldsymbol{\varphi}_{E}$ and $\varphi_{A_{2}}$ transform according to the irreducible representations $E$ and $A_{2}$ respectively, and $\alpha_{\perp}$ and $\alpha_{z}$ are constants $\stackrel{8}{\underline{8}}$ The simplest polynomial expression compatible with the symmetry requirements is

$$
\boldsymbol{\alpha}(\boldsymbol{k})=\alpha_{\perp}\left(k_{y} \hat{x}-k_{x} \hat{y}\right)+\alpha_{z} k_{x} k_{y} k_{z}\left(k_{x}^{2}-k_{y}^{2}\right) \hat{z} .
$$

Setting $\alpha_{z}=0$ here we recover the Rashba model, ${ }^{21}$ which is used to describe the effects of the absence of mirror symmetry in semiconductor quantum wells. In cubic zinc-blende crystals, the momentum dependence of $\boldsymbol{\alpha}(\boldsymbol{k})$ is given by the so-called $k^{3}$, or the Dresselhaus, term. 22.23

One cannot expect Eq. (2) to fully reproduce the spinorbit band splitting in $\mathrm{CePt}_{3} \mathrm{Si}$ and $\mathrm{LaPt}_{3} \mathrm{Si}$, which have quite complicated, multi-sheet, Fermi surfaces. Nevertheless, this expression already captures the most important, symmetry-related, features of the spin-orbit coupling, including the qualitative difference in the $\boldsymbol{k}$ dependences of $\alpha_{x, y}(\boldsymbol{k})$ and $\alpha_{z}(\boldsymbol{k})$, the presence of a band degeneracy line at $k_{x}=k_{y}=0$, and the vanishing of $\alpha_{z}(\boldsymbol{k})$ in the high-symmetry planes. A natural question is whether one can determine the strengths of both the $x y$ - and $z$-components of the spin-orbit coupling using the dHvA experiments.

The eigenvalues of the Hamiltonian (1) are

$$
\epsilon_{\lambda}(\boldsymbol{k})=\epsilon_{0}(\boldsymbol{k})+\lambda\left|\boldsymbol{\alpha}(\boldsymbol{k})-\mu_{B} \boldsymbol{H}\right|,
$$

where $\lambda= \pm$ is the band index (note that the energy bands are split even at $H=0$, if the spin-orbit coupling 
is non-zero). There are two Fermi surfaces determined by the equations

$$
\epsilon_{\lambda}(\boldsymbol{k})=\epsilon_{F},
$$

where $\epsilon_{F}$ is the Fermi energy. Although there may be accidental degeneracies at some magnitudes and directions of the field, in general there are no symmetry reasons for the Fermi surfaces to intersect. Indeed, this would happen if $\boldsymbol{\alpha}(\boldsymbol{k})=\mu_{B} \boldsymbol{H}$. These three equations can have solutions at some isolated points in the first Brillouin zone, which may or may not be on the Fermi surface. The shape of the Fermi surfaces (4) depends on the magnetic field, which can be directly probed by dHvA experiments. In particular, while at $H=0$ we have $\epsilon_{\lambda}(-\boldsymbol{k})=\epsilon_{\lambda}(\boldsymbol{k})$, which is a consequence of time reversal symmetry, in the presence of magnetic field the time-reversal symmetry is lost, and $\epsilon_{\lambda}(-\boldsymbol{k}) \neq \epsilon_{\lambda}(\boldsymbol{k})$, i.e. the Fermi surfaces do not have inversion symmetry, in general.

To calculate the $\mathrm{dHvA}$ frequencies, one needs to include the coupling of the magnetic field to the orbital motion of electrons. In the quasi-classical approximation one can derive the Lifshitz-Onsager quantization rules, ${ }^{20}$ which implicitly determine the energy levels of the band electrons:

$$
S_{\lambda}\left(\epsilon, k_{H}\right)=\frac{2 \pi e H}{\hbar c}\left[n+\gamma_{\lambda}(\Gamma)\right] .
$$

Here $S_{\lambda}$ is the area of the quasi-classical orbit, $\Gamma$, in the $\boldsymbol{k}$ space defined by the intersection of the constant-energy surface $\epsilon_{\lambda}(\boldsymbol{k})=\epsilon$ with the plane $\boldsymbol{k} \cdot \hat{\boldsymbol{H}}=k_{H}(\hat{\boldsymbol{H}}=$ $\boldsymbol{H} / H), n$ is a large integer number, and $0 \leq \gamma_{\lambda}(\Gamma)<1$ is a constant, which depends on the Berry phase acquired by a band electron as it moves along $\Gamma: 24.25$ The value of $\gamma_{\lambda}(\Gamma)$ does not affect the expressions for the dHvA frequencies discussed below.

The oscillating magnetization contains contributions from both bands and can be approximately written as

$$
M_{o s c}=\sum_{\lambda} M_{\lambda} \cos \left(\frac{2 \pi F_{\lambda}}{H}+\phi_{\lambda}\right),
$$

where $M_{\lambda}$ and $\phi_{\lambda}$ are the amplitudes and phases of the oscillations. The expressions for the amplitudes are given by the standard Lifshits-Kosevich formulas ${ }^{20}$ The dHvA frequencies $F_{\lambda}$ are related to the extremal, with respect to $k_{H}$, cross-sectional areas of the two Fermi surfaces as follows

$$
F_{\lambda}=\frac{\hbar c}{2 \pi e} S_{\lambda}^{e x t}
$$

[in addition to the fundamental harmonics (6), the observed dHvA signal also contains higher harmonics with frequencies given by multiple integers of $F_{\lambda}$ ].

If the external field is weak compared to the spin-orbit band splitting, i.e. $\mu_{B} H \ll|\boldsymbol{\alpha}(\boldsymbol{k})|$, the band energies (3) can be represented as a Taylor expansion

$$
\begin{aligned}
\epsilon_{\lambda}(\boldsymbol{k}) & =\epsilon_{0}(\boldsymbol{k})+\lambda|\boldsymbol{\alpha}(\boldsymbol{k})|-\lambda \mu_{B}(\hat{\boldsymbol{\alpha}} \boldsymbol{H}) \\
& +\frac{\lambda \mu_{B}^{2}}{2|\boldsymbol{\alpha}(\boldsymbol{k})|}\left[H^{2}-(\hat{\boldsymbol{\alpha}} \boldsymbol{H})^{2}\right]+\ldots,
\end{aligned}
$$

where $\hat{\boldsymbol{\alpha}}(\boldsymbol{k})=\boldsymbol{\alpha}(\boldsymbol{k}) /|\boldsymbol{\alpha}(\boldsymbol{k})|$. Similarly, the extremal cross-section areas can be written in the form

$$
S_{\lambda}^{\text {ext }}(\boldsymbol{H})=S_{\lambda}^{\text {ext }}(0)+A_{\lambda}(\hat{\boldsymbol{H}}) H+B_{\lambda}(\hat{\boldsymbol{H}}) H^{2}+\ldots
$$

The second, linear in $H$, term on the right-hand side produces the phase shifts in dHvA signal (6). This effect is similar to the usual phase shift due to a paramagnetic splitting of Fermi surfaces in centrosymmetric metals. For some directions of the field, the linear term can be absent, see an example below. The third term and all the subsequent terms produce the magnetic field dependence of the dHvA frequencies. This is a specific feature of the dHvA oscillations in crystals without inversion symmetry, which can be observable if the Zeeman energy is not too small in comparison with spin-orbit coupling. A nonlinear field dependence of the dHvA frequencies has been observed in asymmetric quantum wells $\underline{14}$

To illustrate the above statements, let us look at a simple example of a three-dimensional elliptic Fermi surface with $\epsilon_{0}(\boldsymbol{k})=\hbar^{2} k_{\perp}^{2} / 2 m_{\perp}+\hbar^{2} k_{z}^{2} / 2 m_{z}-\epsilon_{F}$, where $\boldsymbol{k}_{\perp}=\left(k_{x}, k_{y}\right)$, and $m_{\perp}, m_{z}$ are the effective masses. The Fermi momentum $k_{F}$ is introduced via $\epsilon_{F}=\hbar^{2} k_{F}^{2} / 2 m_{\perp}$. We consider only $\boldsymbol{H} \| \hat{z}$ to make connection with the experimental results of Ref. 13, where two main dHvA branches, named $\alpha$ and $\beta$, were detected for this field orientation. One can show that the linear in $H$ terms in the expansions (8) and (9) vanish. The maximum crosssections of the Fermi surfaces correspond to $k_{z}=0$, then $\varphi_{A_{2}}=0$ and we obtain the extremal cross-section area which depends only on the transverse spin-orbit coupling:

$$
S_{\lambda}^{e x t}(\boldsymbol{H})=\pi k_{F}^{2}\left[1-\lambda \frac{\left|\alpha_{\perp}\right| k_{F}}{\epsilon_{F}}\left(1+\frac{\mu_{B}^{2} H^{2}}{2 \alpha_{\perp}^{2} k_{F}^{2}}\right)\right] .
$$

In obtaining this result we used the expression (2) for $\boldsymbol{\alpha}$ and assumed that the Zeeman energy is small compared to the spin-orbit band splitting, which in turn is much smaller than the Fermi energy: $\mu_{B} H \ll\left|\alpha_{\perp}\right| k_{F} \ll \epsilon_{F}$. Although, for a more complicated Fermi surface, there might be additional extremal cross-sections at nonzero $k_{z},{ }^{26}$ the linear in $H$ term in Eq. (9) is still absent due to the symmetry properties of $\varphi_{A_{2}}$.

To estimate the magnitude of the effects under consideration, we use the expressions (77) and (10) to calculate the difference of the dHvA frequencies:

$$
F_{-}-F_{+}=\frac{2 c}{\hbar e}\left|\alpha_{\perp}\right| k_{F} m_{\perp}\left(1+\frac{\mu_{B}^{2} H^{2}}{2 \alpha_{\perp}^{2} k_{F}^{2}}\right) .
$$

The experimental measurement of the splitting of the frequencies allows one to determine the strength of the spin-orbit coupling. Using as an example the frequencies of the $\alpha$ and $\beta$ branches from Ref. $13 F_{\alpha}=1.10 \times 10^{8} \mathrm{Oe}$ and $F_{\beta}=8.41 \times 10^{7} \mathrm{Oe}$, and $m_{\perp} \simeq 1.5 \mathrm{~m}$, we obtain for the spin-orbit splitting of the Fermi surfaces: $\left|\alpha_{\perp}\right| k_{F} \simeq 10^{3} \mathrm{~K}$. While the results of the band structure calculations for $\mathrm{LaPt}_{3} \mathrm{Si}$ reported in Ref. 13 do not 
contain explicit values of the band splitting $\Delta E_{s o}$, for $\mathrm{CePt}_{3} \mathrm{Si}$ one has $\Delta E_{\text {so }} \simeq 50-200 \mathrm{meV}^{\underline{\underline{6}}}$ As for the magnitude of the magnetic field dependence of the frequency splitting, in the range of fields used in Ref. 13 (up to $17 \mathrm{~T})$, we have $\mu_{B} H /\left|\alpha_{\perp}\right| k_{F} \sim 10^{-2}$.

We would like to note that the expansions (8) and therefore (9) fail if $\boldsymbol{\alpha}(\boldsymbol{k})=0$. According to Eqs. (2), this happens if the extremal orbit passes through the poles of the Fermi surface, where the bands are degenerate. In this case, the so-called "magnetic breakdown" occurs, in which the electrons can tunnel from one band to another near the degeneracy points. Instead of Eq. (6), the dHvA signal then contains additional fundamental harmonics corresponding to the quasi-classical orbits switching between different bands $\stackrel{27}{27}$ it not clear if this phenomenon occurs in $\mathrm{LaPt}_{3} \mathrm{Si}$ and $\mathrm{CePt}_{3} \mathrm{Si}$.

In conclusion, we have discussed how the absence of inversion symmetry in the crystal lattice of a metal manifests itself in the $\mathrm{dHvA}$ experiments. The splitting of the $\mathrm{dHvA}$ frequencies is a direct measure of the parameters of the effective spin-orbit Hamiltonian. In particular, according to Eq. (11), it allows one to estimate the magnitude of the "transverse" component of the spin-orbit coupling (in contrast, there seems to be no simple way to determine the $z$-axis component using the dHvA data). Also, the interplay of the Zeeman and the spin-orbit interactions results in a deformation of the Fermi surface, which is responsible for a non-linear field dependence of the dHvA frequencies, the effect absent in centrosymmetric metals.

\section{Acknowledgments}

The authors are grateful to E. I. Rashba for valuable comments and interest to this work. One of us (V.M.) is indebted to Dr. N. Tateiwa for drawing his attention to the recent $\mathrm{dHvA}$ experiments in non-centrosymmetric metals. The support from the Natural Sciences and Engineering Research Council of Canada (K.S.) is gratefully acknowledged.
1 E. Bauer, G. Hilscher, H. Michor, Ch. Paul, E. W. Scheidt, A. Gribanov, Yu. Seropegin, H. Noel, M. Sigrist, and P. Rogl, Phys. Rev. Lett. 92, 027003 (2004).

2 T. Yasuda, H. Shishido, T. Ueda, S. Hashimoto, R. Settai, T. Takeuchi, T. D. Matsuda, Y. Haga, and Y. Onuki, J. Phys. Soc. Japan 73, 1657 (2004).

3 M. Yogi, Y. Kitaoka, S. Hashimoto, T. Yasuda, R. Settai, T. D. Matsuda, Y. Haga, Y. Onuki, P. Rogl, and E. Bauer, Phys. Rev. Lett. 93, 027003 (2004).

4 N. Tateiwa, Y. Haga, T. D. Matsuda, S. Ikeda, T. Yasuda, T. Takeuchi, R. Settai, Y. Onuki, preprint condmat//0505472.

${ }^{5}$ P. A. Frigeri, D. F. Agterberg, A. Koga, and M. Sigrist, Phys. Rev. Lett. 92, 097001 (2004) [Erratum 93, 099903(E) (2004)].

${ }^{6}$ K. V. Samokhin, E. S. Zijlstra, and S. K. Bose, Phys. Rev. B 69, 094514 (2004) [Erratum 70, 069902(E) (2004)].

7 I. A. Sergienko and S. H. Curnoe, Phys. Rev. B 70, 214510 (2004).

8 K. V. Samokhin, Phys. Rev. B 70, 104521 (2004).

${ }^{9}$ V. P. Mineev, Int. J. Mod. Phys. B 18, 2963 (2004).

10 P. A. Frigeri, D. F. Agterberg, and M. Sigrist, New J. Phys. 6, 115 (2004).

11 V. P. Mineev, Phys. Rev. B 71, 012509 (2005).

12 K. V. Samokhin, Phys. Rev. Lett. 94, 027004 (2005).

13 S. Hashimoto, T. Yasuda, T. Kubo, H. Shishido, T. Ueda, R. Settai, T. D. Matsuda, Y. Haga, H. Harima, and Y.
Onuki, J. Phys.: Condens. Matter 16, L287 (2004).

14 J. Luo, H. Munekata, F. F. Fang, and P. J. Stiles, Phys. Rev. B 41, 7685 (1990).

15 E. A. de Andrada e Silva, G. C. La Rocca, and F. Bassani, Phys. Rev. B 50, 8523 (1994).

16 S. A. Tarasenko and N. S. Averkiev, Pis'ma Zh. Eksp. Teor. Fiz 75, 669 (2002) [JETP Lett. 75, 552 (2002)].

17 M. M. Miller and R. Reifenberger, Phys. Rev. B 38, 4120 (1988).

18 I. I. Boiko and E. I. Rashba, Fiz. Tverd. Tela (Leningrad) 2, 1874 (1960) [Sov. Phys. Solid State 2, 1692 (1960)].

19 X. Wang, P. Vasilopoulos, Phys. Rev. B 72, 085344 (2005).

20 E. M. Lifshitz and L. P. Pitaevskii, Statistical Physics, Part 2 (Butterworth-Heinemann, Oxford, 1995).

21 E.I.Rashba, Fiz. Tverd. Tela (Leningrad) 2, 1224 (1960) [Sov. Phys. Solid State 2, 1109 (1960)].

22 G. Dresselhaus, Phys. Rev. 100, 580 (1955).

23 L. M. Roth, Phys. Rev. 173, 755 (1968).

24 G. P. Mikitik and Yu. V. Sharlai, Phys. Rev. Lett. 82, 2147 (1999).

25 F. D. M. Haldane, Phys. Rev. Lett. 93, 206602 (2004).

${ }^{26}$ One can show that, in addition to the plane $k_{z}=0, \alpha_{z}(\boldsymbol{k})$ also vanishes on the horizontal faces of the first Brillouin zone, i.e. $k_{z}= \pm \pi / c$.

27 D. Shoenberg, Magnetic Oscillations in Metals (Cambridge University Press, Cambridge, 1984). 